\title{
Role of the E3 ubiquitin ligase gene related to anergy in lymphocytes in glucose and lipid metabolism in the liver
}

\author{
Sachie Nakamichi ${ }^{1}$, Yoko Senga ${ }^{1}$, Hiroshi Inoue ${ }^{2}$, Aki Emi $^{1}$, Yasushi Matsuki ${ }^{3}$, \\ Eijiro Watanabe $^{3}$, Ryuji Hiramatsu ${ }^{3}$, Wataru Ogawa ${ }^{1}$ and Masato Kasuga ${ }^{1,4}$ \\ ${ }^{1}$ Division of Diabetes, Metabolism, and Endocrinology, Department of Internal Medicine, Kobe University Graduate School of Medicine, \\ 7-5-1 Kusunoki-cho, Chuo-ku, Kobe 650-0017, Japan \\ ${ }^{2}$ Frontier Science Organization, Kanazawa University, Kanazawa 920-8641, Japan \\ ${ }^{3}$ Pharmacology Research Laboratories, Dainippon Sumitomo Pharmaceuticals Co. Ltd, Osaka 554-0022, Japan \\ ${ }^{4}$ Research Institute, International Medical Center of Japan, Tokyo 162-8655, Japan \\ (Correspondence should be addressed to W Ogawa; Email: ogawa @med.kobe-u.ac.jp)
}

\begin{abstract}
Gene related to anergy in lymphocytes (GRAIL) is an E3 ubiquitin ligase that regulates energy in T-lymphocytes. Whereas, the relevance of GRAIL to T lymphocyte function is well established, the role of this protein in other cell types remains unknown. Given that GRAIL is abundant in the liver, we investigated the potential function of GRAIL in nutrient metabolism by generating mice in which the expression of GRAIL is reduced specifically in the liver. Adenovirusmediated transfer of a short hairpin RNA specific for GRAIL mRNA markedly reduced the amounts of GRAIL mRNA and protein in the liver. Blood glucose levels of the mice with hepatic GRAIL deficiency did not differ from those of control animals in the fasted or fed states. However, these mice manifested glucose intolerance in association with a normal increase in plasma insulin levels during glucose challenge. The mice also manifested an increase in the serum concentration of free fatty acids, whereas the serum levels of cholesterol and triglyceride were unchanged. The hepatic abundance of mRNAs for glucose-6-phosphatase, catalytic (a key enzyme in hepatic glucose production) and for sterol regulatory element-binding transcription factor 1 (an important transcriptional regulator of lipogenesis) was increased in the mice with hepatic GRAIL deficiency, possibly contributing to the metabolic abnormalities of these animals. Our results thus demonstrate that GRAIL in the liver is essential for maintenance of normal glucose and lipid metabolism in living animals.
\end{abstract}

Journal of Molecular Endocrinology (2009) 42, 161-169

\section{Introduction}

The liver plays a central role in nutrient metabolism. It stores carbohydrate as glycogen in the fed state, and it releases glucose, produced either through the breakdown of glycogen (glycogenolysis) or through de novo synthesis (gluconeogenesis), into the circulation in the fasted state. The liver is also the major site for the synthesis and oxidation of fatty acids as well as a source of secreted triglyceride and cholesterol. Dysregulation of nutrient metabolism in the liver thus results in various disorders including type 2 diabetes mellitus, dyslipidemia, atherosclerosis, and fatty liver disease, all of which have become global health problems.

Gene related to anergy in lymphocytes (GRAIL, also known as the product of the gene Rnf128) is a transmembrane RING finger-type E3 ubiquitin ligase. The abundance of GRAIL mRNA is increased in T-lymphocytes in response to the induction of anergy (Anandasabapathy et al. 2003), and the encoded protein plays an important role in the regulation of anergy in these cells (Seroogy et al. 2004, Soares et al. 2004, Lineberry et al. 2008a) by catalyzing the ubiquitination of target proteins including CD40 (Lineberry et al. 2008a) as well as CD81 and CD151 (Lineberry et al. 2008b). Although the expression of GRAIL in T-lymphocytes correlates well with the function of these cells (Heissmeyer et al. 2004), GRAIL is expressed in various other cell types and organs (Anandasabapathy et al. 2003). The physiological relevance of this protein in cells other than T-lymphocytes has remained unknown, however.

Given that, among major organs, the abundance of GRAIL mRNA is greatest in the liver (Anandasabapathy et al. 2003), we have investigated the function of GRAIL in this organ. With the use of adenovirus-mediated transfer of short hairpin RNA (shRNA), a technique that has been widely used to investigate the function of specific proteins in the liver (Koo et al. 2004, 2005, Taniguchi et al. 2005), we have generated mice in which 
the abundance of GRAIL is reduced specifically in the liver. We now present evidence that GRAIL in the liver is required for maintenance of normal glucose and lipid metabolism in living animals.

\section{Materials and methods}

\section{Antibodies to GRAIL}

A cDNA encoding the RING-finger domain of mouse GRAIL (amino acids 231-421) was synthesized with the use of PCR and cloned into the pGEX4T-1 vector (GE Healthcare, Amersham,UK) for expression of a glutathione S-transferase (GST) fusion protein. Polyclonal antibodies to GRAIL were generated by injection of rabbits with the GST-GRAIL fusion protein. For the analysis of GRAIL expression in various tissues, tissue extracts $(100 \mu \mathrm{g}$ protein) prepared from 15-week-old male $\mathrm{C} 57 \mathrm{BL} / 6$ mice were subjected to immunoblot analysis.

\section{Expression vectors for mouse GRAIL}

A cDNA encoding full-length mouse GRAIL was isolated by PCR and ligated into pcDNA3.1(+; Invitrogen). A mutant GRAIL cDNA in which $\mathrm{C}$ residues at nucleotide positions 888 and 897 of the open reading frame were replaced by A was generated with the use of a QuickChange Site-Directed Mutagenesis Kit (Stratagene, La Jolla, CA, USA).

\section{Constructs encoding GRAIL shRNAs}

The nucleotide sequence of mouse GRAIL mRNA was analyzed for the generation of shRNAs according to the rational design rules (Reynolds et al. 2004). Three complementary antiparallel oligonucleotides corresponding to nucleotides 325-343, 873-891, and 10691087 of the open reading frame were synthesized together with a loop sequence (acgtgtgctgtccgt). The respective oligonucleotides synthesized were thus as follows: GSX1, gtttGGGGCATGGTGTAAGTATAacgtgt gctgtccgtTATACTTGCACCGTGCTCCttttt (forward) and atgcaaaaaGGAGCACGGTGCAAGTATAacggacagcacacgtTATACTTACACCATGCCCC (reverse); GSX2, gtttATTCTAGCCTGCAATTATAacgtgtgctgtccgtTATGATTGCAGGTTAGGATttttt (forward) and atgcaaaaaATC-CTAACCTGCAATCATAacggacagcacacgtTATAATTGCAGGCTAGAAT (reverse); and GSX3, gtttGTTGTAGTGAGACTGTATCacgtgtgctgtccgtGATGCAGTCTCACTGCGACttttt (forward) and atgcaaaaaGTCGCAGTGA-GACTGCATCacggacagcacacgtGATACAGTCTCACTACAAC (reverse). The forward and reverse oligonucleotides were annealed and then ligated into the pcPURmU6icassette vector (Takara Bio, Ohtsu,
Japan), which contains the mouse U6 promoter. For screening of the efficacy and specificity of the shRNA constructs, COS7 cells were transfected both with the pcPURmU6icassette vectors encoding GSX1, -2 , or -3 shRNAs and with pcDNA3.1(+) encoding wild-type or mutant GRAIL with the use of the lipofectamine reagent (Invitrogen). Lysates of the transfected cells were then subjected to immunoblot analysis with antibodies to GRAIL. For production of adenovirus vectors encoding the GSX2 shRNA or containing the U6 promoter alone, the GSX2 sequence together with the U6 promoter or the U6 promoter alone was excised from the corresponding pcPURmU6icassette vector and ligated into the pAxcwit cosmid cassette (Takara Bio). The adenoviruses encoding GRAIL shRNA (AxshGRAIL) or containing the U6 promote alone (AxU6) were then generated with the use of an Adenovirus Expression Vector Kit (Takara Bio) as described previously (Kitamura et al. 1999).

\section{Generation and metabolic analysis of mice with liver-specific deficiency of GRAIL}

All animal procedures were performed in accordance with the guidelines of the animal ethics committee of Kobe University Graduate School of Medicine. Eightweek-old male C57BL/6 mice were injected through the tail vein with AxshGRAIL or AxU6 at a dose of $1 \times$ $10^{9}$ plaque-forming units per $30 \mathrm{~g}$ body mass. Mice were subjected to various assays 4 days after adenoviral injection unless indicated otherwise. For a glucose tolerance test (GTT), mice deprived of food for $16 \mathrm{~h}$ were loaded intraperitoneally with glucose $(2 \mathrm{~g} / \mathrm{kg})$. Blood glucose and plasma insulin concentrations were measured as described (Miyake et al. 2002). Serum levels of triglyceride, cholesterol, free fatty acids, and alanine aminotransferase were determined with the use of a Triglyceride $E$-test, cholesterol $E$-Test, NEFA $C$-test, and transaminase CII-test (Wako, Osaka, Japan) respectively in animals deprived of food for $16 \mathrm{~h}$. The amounts of mRNAs derived from various genes in the liver were also determined in mice that had been deprived of food for $16 \mathrm{~h}$.

\section{Gene expression analysis}

Reverse transcription (RT) and real-time PCR analysis with 36B4 mRNA as the invariant control was performed as described (Miyake et al. 2002). The primers for glucose-6-phosphatase, catalytic (G6pc), peroxisome proliferator-activated receptor gamma, coactivator 1 alpha (PPARGC1A; Inoue et al. 2006), phosphoenolpyruvate carboxykinase 1, cytosolic (PCK1; Teshigawara et al. 2005), fatty acid synthase (FAS; Okamoto et al. 2007), and sterol regulatory elementbinding transcription factor 1 (SREBF1; Matsumoto et al. 
2003) were as described. The primers for mouse GRAIL were $5^{\prime}$-GGGAGCACGGTGCAAGTATC-3' (forward) and $5^{\prime}$-TTCCTTTCAGATTGCCAATCAT-3 ${ }^{\prime}$ (reverse). The primers for mouse CREB regulated transcription coactivator 2 (CRTC2) were obtained from QIAGEN (QuantiTect Primer Assay, Valencia, CA, USA). For northern blot analysis, a membrane loaded with $20 \mu \mathrm{g}$ total RNA extracted from various mouse organs (Mouse Tissue Total RNA Northern Blot; BioChain, Hayward, CA, USA) was probed with a ${ }^{32} \mathrm{P}$-labeled fragment of mouse GRAIL cDNA (nucleotides 138-482) isolated by PCR. For assay of hepatic gene expression during refeeding, C57BL/6 mice were deprived of food for $24 \mathrm{~h}$ and then fed with a high-carbohydrate chow containing $70 \%$ sucrose.

\section{Statistical analysis}

Unless indicated otherwise, quantitative data are presented as means \pm s.E.M. Differences between groups were evaluated by Student's $t$-test. A $P<0.05$ was considered statistically significant.

\section{Results}

\section{Tissue distribution of GRAIL}

Northern blot analysis revealed that GRAIL mRNA was most abundant in heart, kidney, and liver, and was present in smaller amounts in brain, skeletal muscle, and white adipose tissue (Fig. 1A), essentially consistent with the results of a previous study (Anandasabapathy et al. 2003). Although GRAIL mRNA was not detected in lung and spleen by northern analysis (Fig. 1A), it was found to be present in small amounts in these two organs as well as in testis by RT and real-time PCR analysis (Fig. 1B). GRAIL mRNA was also detected in epididymal and brown adipose tissue, but not in s.c. adipose tissue, by RT and real-time PCR analysis. Immunoblot analysis with antibodies to GRAIL revealed that, among major mouse organs, GRAIL protein was most abundant in the liver (Fig. 1C). GRAIL protein was glycosylated in cells and thus observed as multiple bands (Anandasabapathy et al. 2003). The difference in apparent molecular weight of GRAIL protein in multiple tissues might be attributable to the difference in the extent of glycosylation. The abundance of GRAIL mRNA was significantly suppressed after refeeding whereas that of SREBF1 was increased in the liver of mice (Fig. 1D).

\section{Generation and specificity of GRAIL shRNAs}

Systemic infusion of adenovirus vectors results in the liver-specific expression of exogenous genes (Miyake
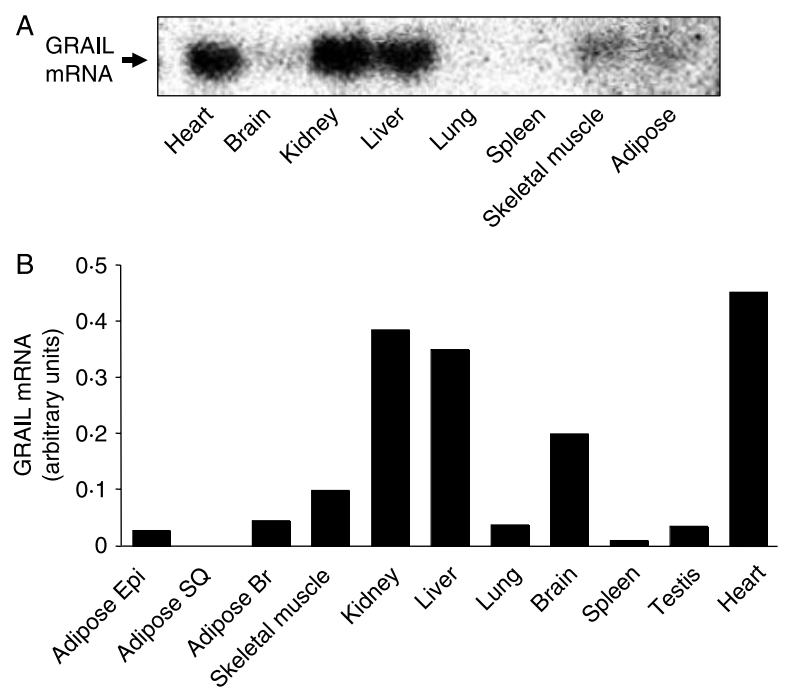

C
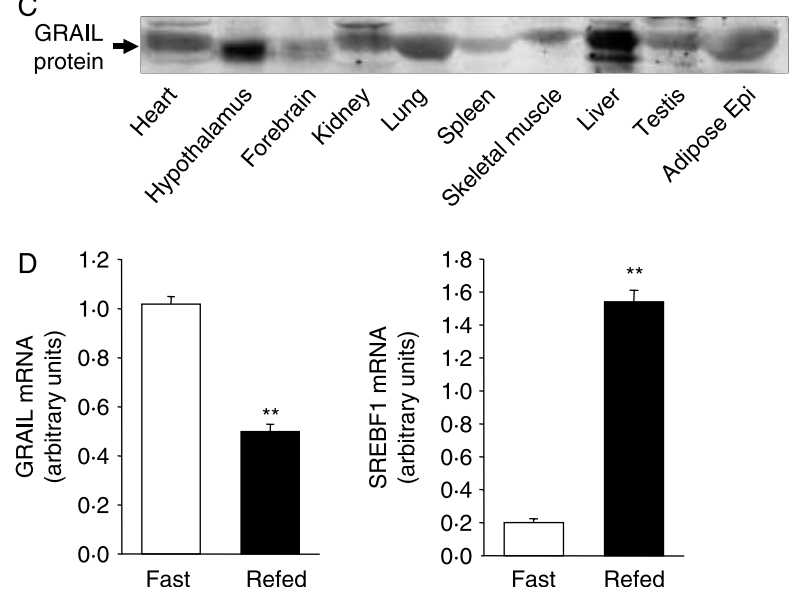

Figure 1 Tissue distribution of GRAIL. (A) Northern blot analysis of GRAIL mRNA in various mouse organs. (B) Total RNA isolated from the indicated organs of 15-week-old male C57BL/6 mice $(n=5)$ in the randomly fed state was separately pooled and subjected to RT and real-time PCR analysis of GRAIL mRNA. Adipose Epi, epididymal adipose tissue; Adipose SQ, s.c. adipose tissue; Adipose $\mathrm{Br}$, brown adipose tissue. Data are means of duplicate experiments. (C) Tissue extracts prepared from C57BL/6 mice in the randomly fed state were subjected to immunoblot analysis with antibodies to GRAIL. (D) Expression of GRAIL mRNA in the liver of C57BL/6 mice at the fasted and the refed states. Data are means \pm S.E.M. of seven experiments; ${ }^{\star *} P<0.01$ versus the fasted states (Student's $t$-test).

et al. 2002), and adenovirus-mediated introduction of shRNAs induces a specific reduction in the amount of the target protein in the liver (Koo et al. 2004, 2005, Taniguchi et al. 2005). To explore the function of GRAIL in the liver, we therefore attempted to downregulate the expression of GRAIL in this organ by adenovirus-mediated introduction of shRNA in mice. We generated three shRNA constructs (GSX1, GSX2, and GSX3) targeted to mouse GRAIL mRNA (Fig. 2A). 
Vectors encoding each of these constructs were introduced individually into COS7 cells by transfection together with an expression vector encoding wild-type mouse GRAIL. Although all three constructs inhibited the expression of GRAIL protein, the effect of GSX2 was most pronounced (Fig. 2B). To verify the specificity of the shRNA constructs, we next tested their effects on the expression of a mutant form of GRAIL in which a single nucleotide in the target sequence of GSX2 was altered (Fig. 2A). GSX1 and GSX3 exhibited relatively small inhibitory effects on the expression of the GRAIL mutant (Fig. 2C) as they did on that of the wild-type protein (Fig. 2B). However, the inhibitory effect of GSX2 was almost completely abolished (Fig. 2C), indicating the high specificity of GSX2 for its target sequence in GRAIL mRNA. For subsequent experiments, we therefore focused on the effects of an adenovirus vector encoding GSX2 (AxshGRAIL). To examine whether GSX2 inhibits the expression of endogenous GRAIL, we infected cultured AML12 mouse hepatocytes with AxshGRAIL or a control adenovirus containing the $\mathrm{U} 6$ promoter alone

A

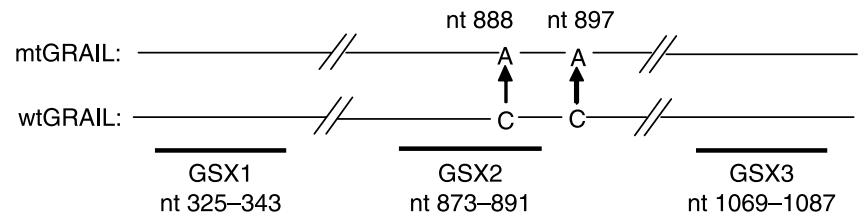

B

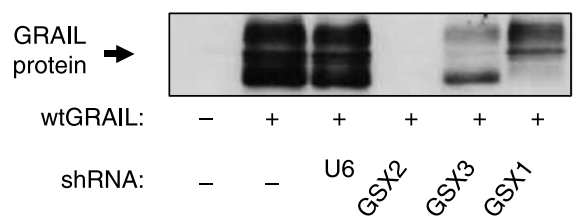

C

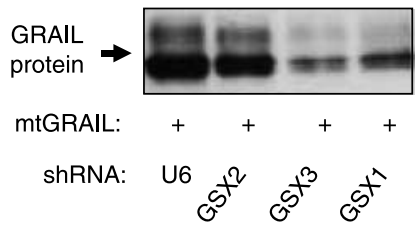

D

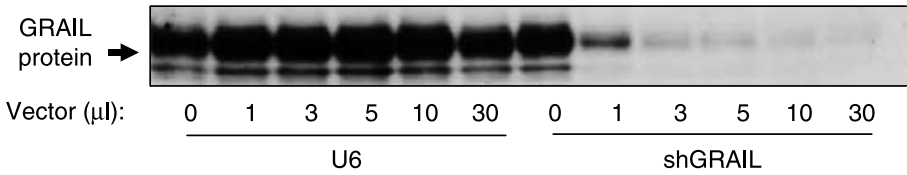

E

GRAIL protein

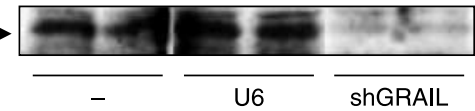

\section{G}

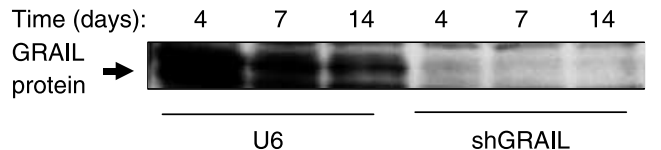

F

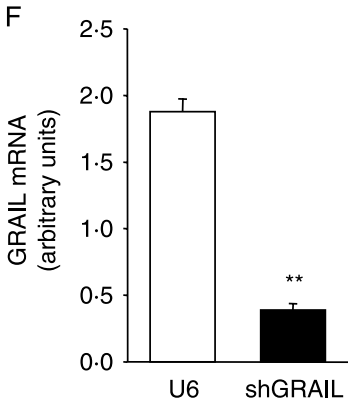

Figure 2 Efficiency and specificity of GRAIL shRNAs. (A) Schematic of wild-type (wt) and mutant (mt) GRAIL mRNAs and of the shRNA constructs. (B and C) COS7 cells cultured in $6 \mathrm{~cm}$ plates were transfected with $1.5 \mu \mathrm{g}$ pcDNA3.1 $(+)$ encoding wild-type (B) or mutant (C) GRAIL as well as with $1.5 \mu \mathrm{g}$ pcPURmU6icassette either encoding GSX1, GSX2, or GSX3 GRAIL shRNAs or containing the U6 promoter alone, as indicated. The cells were then subjected to immunoblot analysis with antibodies to GRAIL. (D) AML12 cells cultured in six-well plates were infected for $48 \mathrm{~h}$ with the indicated volumes of AxshGRAIL or AxU6 at a concentration of $1 \times 10^{10} \mathrm{PFU} / \mathrm{ml}$. The cells were then lysed and subjected to immunoblot analysis with antibodies to GRAIL. (E-G) C57BL/6 mice were injected or not (-) with AxshGRAIL or AxU6 at a dose of $1 \times 10^{9} \mathrm{PFU} / 30 \mathrm{~g}$. The liver was removed and subjected to immunoblot analysis with antibodies to GRAIL ( $E$ and $G$ ) or to RT and real-time PCR analysis of GRAIL mRNA (F) either 4 days ( $E$ and $F$ ) or the indicated times $(G)$ after adenoviral infection. All immunoblots are representative of two or three experiments, and the data in (F) are means \pm S.E.M. of 11 experiments; ${ }^{\star \star} P<0.01$ versus U6 (Student's $t$-test). 
(AxU6). Immunoblot analysis of cell lysates showed that infection of the cells with AxshGRAIL reduced the amount of endogenous GRAIL protein in a dosedependent manner, whereas AxU6 had no such effect (Fig. 2D).

\section{Specific depletion of GRAIL in mouse liver}

We infected 8-week-old male C57BL/6 mice with AxshGRAIL or AxU6 through injection into the tail vein, and we subjected the animals to various assays 4 days after the injection. As expected, infection of mice with AxshGRAIL markedly reduced the hepatic abundance of GRAIL protein whereas that with AxU6 did not (Fig. 2E). RT and real-time PCR analysis revealed that infection with AxshGRAIL reduced the hepatic abundance of GRAIL mRNA by $\sim 80 \%$ compared with that in mice infected with AxU6 (Fig. 2F). The marked reduction in the amount of GRAIL protein in the liver persisted for at least 14 days after AxshGRAIL infection (Fig. 2G). Serum levels of alanineaminotransferase, a marker of liver damage, were similar in mice injected with AxshGRAIL or AxU6 and were within normal limits in both groups of mice $(17.85 \pm 0.59$ and $17.93 \pm 1.15 \mathrm{IU} / 1$ respectively, $n=5$ ) indicating that neither adenoviral infection at the selected dose nor the $\sim 80 \%$ reduction in the level of GRAIL expression induced liver damage.

\section{Metabolic alterations in mice with liver-specific deficiency of GRAIL}

Given that the liver plays a central role in nutrient metabolism, we investigated various parameters of glucose and lipid metabolism in mice with liver-specific depletion of GRAIL. Body mass in the fasted (21.2 \pm 0.22 and $21 \cdot 2 \pm 0.41 \mathrm{~g}, n=5)$ or randomly fed $(23.5 \pm$ $0 \cdot 24$ and $23 \cdot 4 \pm 0 \cdot 18 \mathrm{~g}, n=5)$ states did not differ between mice injected with or AxU6 (respectively). Although blood glucose levels in the fasted (Fig. 3A) or randomly fed (Fig. 3B, AxshGRAIL) states were similar in the two groups of mice, the increase in those during a GTT was exaggerated in mice injected with AxshGRAIL (AxshGRAIL, Fig. 3C). Plasma insulin levels during the GTT did not differ significantly, however, between the two groups of mice (Fig. 3D). Whereas, the serum concentrations of cholesterol and triglyceride were similar in the two groups of mice, the serum concentration of free fatty acids was greater in mice injected with than in those injected with AxU6 (Fig. 3E).

\section{Changes in hepatic gene expression in mice with liver-specific deficiency of GRAIL}

Regulation of hepatic gene expression is largely responsible for the control of both glucose and lipid metabolism (O'Brien et al. 2001, Shimano 2001). We therefore investigated hepatic gene expression in mice infected with AxshGRAIL. The abundance of mRNAs for G6pc, an important enzyme in hepatic glucose production (Radziuk \& Pye 2001), and for SREBF1, a transcription factor that regulates the expression of various lipogenic genes (Shimano 2001), was increased in mice injected with AxshGRAIL compared with that in those injected with AxU6 (Fig. 4). The amounts of mRNAs for PCK1, a rate-limiting enzyme for gluconeogenesis (Radziuk \& Pye 2001), and for FAS, an enzyme that contributes to fatty acid synthesis (Shimano 2001), were both slightly increased by infection with AxshGRAIL, but these changes were not statistically significant. PPARGC1A and CRTC2 are transcriptional coactivators that contribute to regulation of gluconeogenic genes (Herzig et al. 2001, Yoon et al. 2001, Koo et al. 2005). The mRNA abundance of PPARGC1A as well as of CRTC2 was not affected by infection with AxshGRAIL.

\section{Discussion}

With the use of adenovirus-mediated transfer of shRNA, we have generated a mouse model in which the expression of GRAIL is acutely reduced in the liver. These animals manifested an exaggerated increase in blood glucose levels in response to glucose challenge. Given that the associated increase in plasma insulin levels of these mice was similar to that of control mice, the glucose intolerance induced by hepatic GRAIL deficiency was likely attributable not to a defect in insulin secretion but to impairment of insulin sensitivity. The dysregulation of hepatic glucose production is an important pathological feature of type 2 diabetes (DeFronzo 2004), and the control of hepatic glucose production is thought to be largely dependent on the abundance of enzymes that mediate this process (Radziuk \& Pye 2001). Indeed, forced expression of G6pc or PCK1 in the liver induces frank glucose intolerance in rodents (Valera et al. 1994, Trinh et al. 1998). It is thus likely that the observed increase in the abundance of G6pc mRNA in the liver contributes to the glucose intolerance of mice with hepatic deficiency of GRAIL.

The expression of genes involved in hepatic glucose production is controlled by an elaborate network of transcriptional regulators. Such regulators include the transcription factors $c A M P$ response element-binding protein (CREB), forkhead box O1 (FoxO1), hepatocyte nuclear factor 4 alpha (HNF4 $\alpha$ ), signal transducer and activator of transcription 3 (STAT3), and Krüppel-like factor 15 (KLF15; Yoon et al. 2001, Teshigawara et al. 2005, Inoue et al. 2006, Gross et al. 2008) as well as the transcriptional coactivators CREB-binding protein 
A

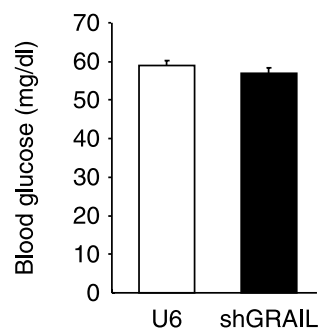

C

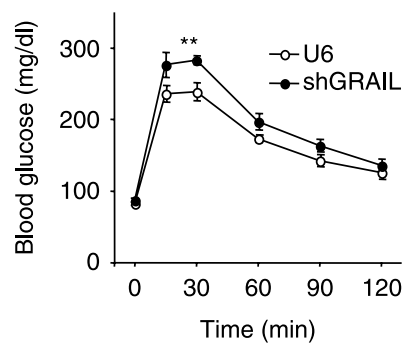

D

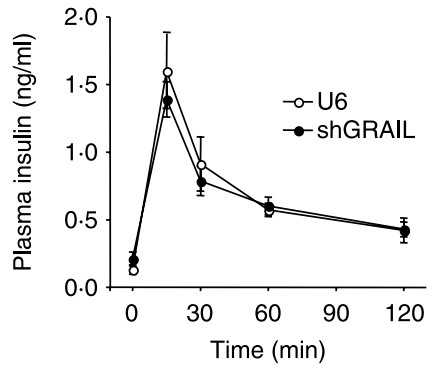

B
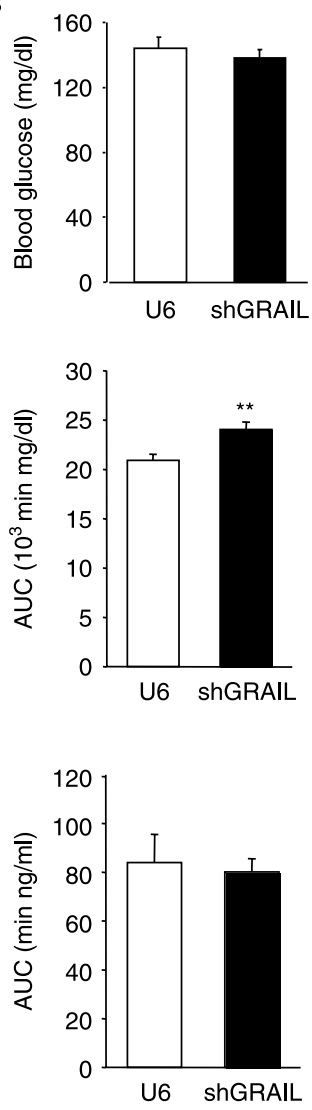
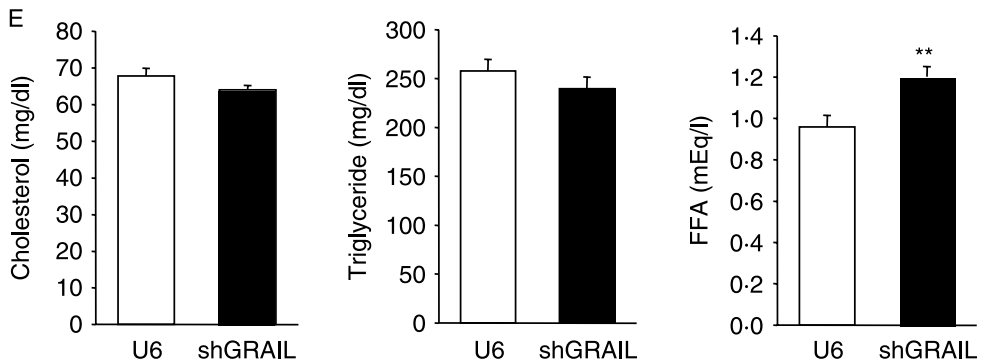

Figure 3 Metabolic characteristics of mice with liver-specific deficiency of GRAIL ( $A$ and $B$ ) Blood glucose concentrations in the fasted $(A)$ or randomly fed (B) states of mice injected with AxshGRAIL or AxU6. (C and D) Blood glucose (C) and plasma insulin (D) concentrations during a GTT in mice injected with AxshGRAIL or AxU6. Values at various times after glucose administration are shown in the left panels, and the area under the curve (AUC) during the test is shown in the right panels. (E) Serum concentrations of cholesterol, triglyceride, and free fatty acids (FFA) in the fasted state of mice injected with AxshGRAIL or AxU6. Data are means \pm S.E.M. of values from 5 ( $A$ and $E$ ), $8(B)$, or 13 and 11 (shGRAIL and $\mathrm{U} 6$ respectively in (C) and (D)) mice. ${ }^{\star \star} P<0.01$ versus U6 (Student's $t$-test).

(CREBBP), PPARGC1A, and CRTC2 (Herzig et al. 2001, Yoon et al. 2001, Koo et al. 2005, Gross et al. 2008). Moreover, several nuclear receptors including liver X receptor (Cao et al. 2003), farnesoid X receptor (Zhang et al. 2006), and small heterodimer partner (Kim et al.
2008) also appear to contribute to the regulation of the expression of genes for hepatic glucose production. Evidence suggests that the activity, abundance, or subcellular distribution of certain transcriptional regulators is influenced by ubiquitination(Huang et al. 

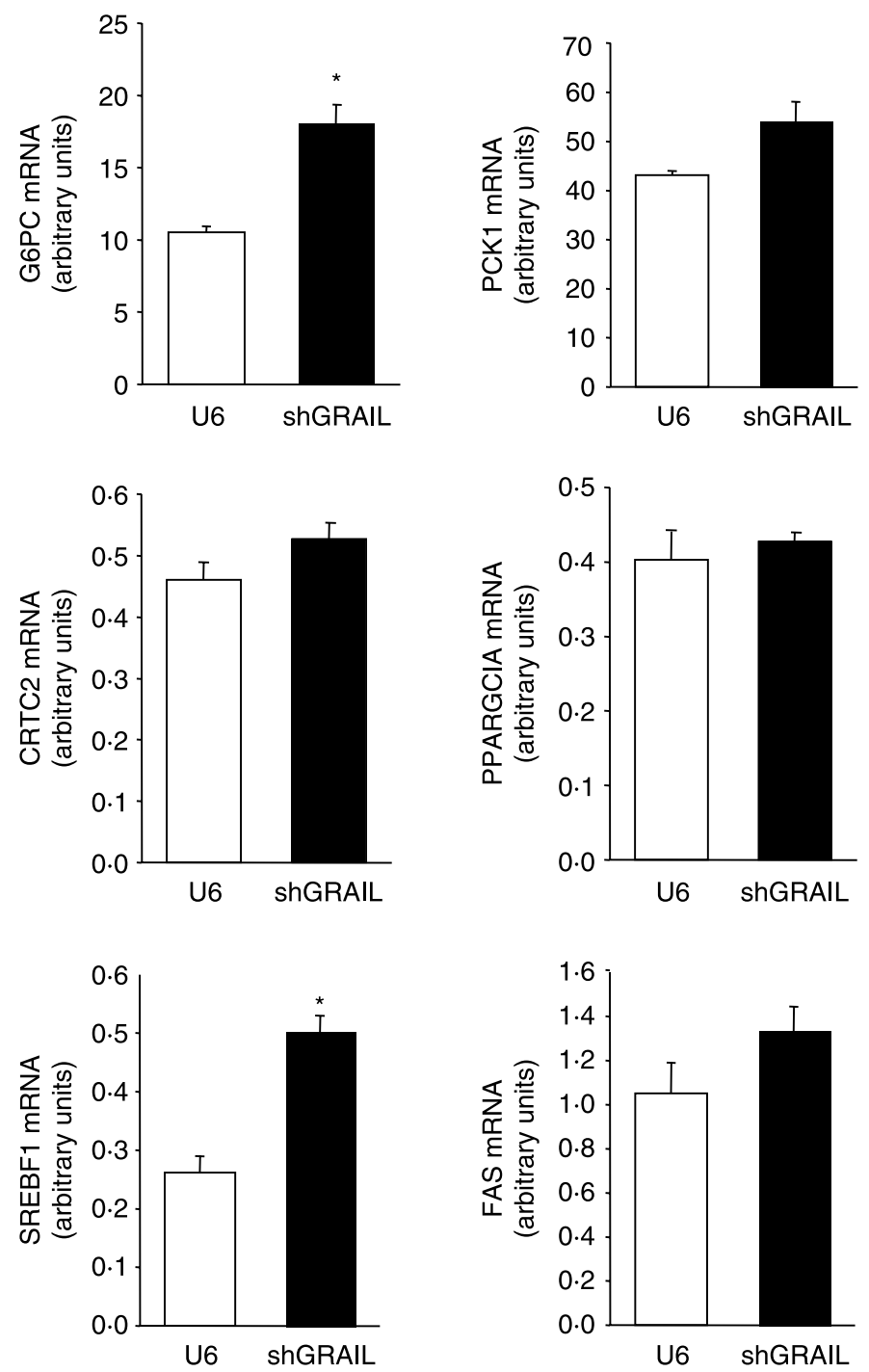

Figure 4 Changes in hepatic gene expression in mice with liver-specific deficiency of GRAIL. Total RNA isolated from the liver of fasted mice injected with AxshGRAIL or AxU6 was separately combined and subjected to RT and real-time PCR analysis of the indicated mRNAs. Data are means \pm S.E.M. from three experiments each performed with total RNA pooled from five mice per group. ${ }^{*} P<0.05$ versus U6

(Student's $t$-test).

2005, Chen et al. 2007, Dentin et al. 2007, Sano et al. 2007, Kato et al. 2008). GRAIL might also affect the expression of genes related to glucose production through the ubiquitination of such transcriptional regulators. Although the mRNA abundance of PPARGC1A and CRTC2 in mouse liver was not affected by shRNA of GRAIL, we cannot exclude the possibility that GRAIL affects the protein abundance of these molecules.

Another metabolic alteration induced by depletion of GRAIL in the liver was an increase in the serum concentration of free fatty acids. Given that an acute increase in the circulating concentration of free fatty acids results in insulin resistance in both humans and rodents (Savage et al. 2007), this defect in lipid metabolism might also be related to the glucose intolerance in these mice. SREBF1 is a transcription factor that controls the expression of several genes that encode enzymes involved in fatty acid synthesis (Shimano 2001). The observed increase in the abundance of SREBF1 mRNA in the liver of mice with hepatic GRAIL deficiency is thus consistent with the 
increase in the serum level of free fatty acids. Furthermore, the mRNA abundance of hepatic GRAIL was suppressed after refeeding whereas that of SREBF1 was increased. This observation is consistent with a notion that GRAIL contributes to negative regulation of SREBF1. The abundance of FAS mRNAs which is regulated by SREBF1 (Shimano 2001), was slightly greater in the liver of mice with hepatic GRAIL deficiency than in that of control mice, although this difference did not achieve statistical significance.

In summary, by generating mice with hepatic GRAIL deficiency, we have shown that this E3 ubiquitin ligase in the liver is important for normal glucose and lipid metabolism in living animals. Our study has thus uncovered a physiological role for GRAIL in a cell type other than T-lymphocytes. The mechanism by which GRAIL influences the expression of genes in the liver remains to be elucidated. Further investigation of this mechanism may shed light on the pathophysiology of metabolic diseases such as type 2 diabetes or dyslipidemia.

\section{Declaration of interest}

There is no conflict of interest that could be perceived as prejudicing the impartiality of the research reported.

\section{Funding}

This work was supported by grants from the Ministry of Education, Culture, Sports, Science, and Technology of Japan (MEXT) to M Kasuga and W Ogawa; a grant for the 21st Century COE Program 'Center of Excellence for Signal Transduction Disease: Diabetes Mellitus as Model' from MEXT to M Kasuga; a grant for the Cooperative Link of Unique Science and Technology for Economy Revitalization (CLUSTER) from MEXT to M Kasuga; and a grant from Takeda Medical Foundation to W Ogawa.

\section{References}

Anandasabapathy N, Ford GS, Bloom D, Holness C, Paragas V, Seroogy C, Skrenta H, Hollenhorst M, Fathman CG \& Soares L 2003 GRAIL: an E3 ubiquitin ligase that inhibits cytokine gene transcription is expressed in anergic CD4+ T cells. Immunity 18 535-547.

Cao G, Liang Y, Broderick CL, Oldham BA, Beyer TP, Schmidt RJ, Zhang Y, Stayrook KR, Suen C, Otto KA et al. 2003 Antidiabetic action of a liver $\mathrm{X}$ receptor agonist mediated by inhibition of hepatic gluconeogenesis. Journal of Biological Chemistry $\mathbf{2 7 8}$ 1131-1136.

Chen J, Halappanavar S, Th'ng JP \& Li Q 2007 Ubiquitin-dependent distribution of the transcriptional coactivator p300 in cytoplasmic inclusion bodies. Epigenetics 2 92-99.

DeFronzo RA 2004 Pathogenesis of type 2 diabetes mellitus. Medical Clinics of North America $\mathbf{8 8}$ 787-835.

Dentin R, Liu Y, Koo SH, Hedrick S, Vargas T, Heredia J, Yates J III \& Montminy M 2007 Insulin modulates gluconeogenesis by inhibition of the coactivator TORC2. Nature 449 366-369.

Gross DN, van den Heuvel AP \& Birnbaum MJ 2008 The role of FoxO in the regulation of metabolism. Oncogene 27 2320-2336.
Heissmeyer V, Macian F, Im SH, Varma R, Feske S, Venuprasad K, Gu H, Liu YC, Dustin ML \& Rao A 2004 Calcineurin imposes T cell unresponsiveness through targeted proteolysis of signaling proteins. Nature Immunology 5 255-265.

Herzig S, Long F, Jhala US, Hedrick S, Quinn R, Bauer A, Rudolph D, Schutz G, Yoon C, Puigserver P et al. 2001 CREB regulates hepatic gluconeogenesis through the coactivator PGC-1. Nature 413 179-183.

Huang H, Regan KM, Wang F, Wang D, Smith DI, van Deursen JM \& Tindall DJ 2005 Skp2 inhibits FOXO1 in tumor suppression through ubiquitin-mediated degradation. PNAS 102 1649-1654.

Inoue H, Ogawa W, Asakawa A, Okamoto Y, Nishizawa A, Matsumoto M, Teshigawara K, Matsuki Y, Watanabe E, Hiramatsu R et al. 2006 Role of hepatic STAT3 in brain-insulin action on hepatic glucose production. Cell Metabolism 3 267-275.

Kato S, Ding J, Psick E, Jhala US \& Du K 2008 COP1 functions as a FoxO1 ubiquitin E3 ligase to regulate FoxO1-mediated gene expression. Journal of Biological Chemistry 283 35464-35473.

Kim YD, Park KG, Lee YS, Park YY, Kim DK, Nedumaran B, Jang WG, Cho WJ, Ha J, Lee IK et al. 2008 Metformin inhibits hepatic gluconeogenesis through AMP-activated protein kinase-dependent regulation of the orphan nuclear receptor SHP. Diabetes 57 306-314.

Kitamura T, Kitamura Y, Kuroda S, Hino Y, Ando M, Kotani K, Konishi H, Matsuzaki H, Kikkawa U, Ogawa W et al. 1999 Insulin-induced phosphorylation and activation of cyclic nucleotide phosphodiesterase 3B by the serine-threonine kinase Akt. Molecular and Cellular Biology 19 6286-6296.

Koo SH, Satoh H, Herzig S, Lee CH, Hedrick S, Kulkarni R, Evans RM, Olefsky J \& Montminy M 2004 PGC-1 promotes insulin resistance in liver through PPAR- $\alpha$-dependent induction of TRB-3. Nature Medicine 10 530-534.

Koo SH, Flechner L, Qi L, Zhang X, Screaton RA, Jeffries S, Hedrick S, $\mathrm{Xu}$ W, Boussouar F, Brindle P et al. 2005 The CREB coactivator TORC2 is a key regulator of fasting glucose metabolism. Nature 437 1109-1111.

Lineberry NB, Su LL, Lin JT, Coffey GP, Seroogy CM \& Fathman CG $2008 a$ The transmembrane E3 ligase GRAIL ubiquitinates the costimulatory molecule CD40 ligand during the induction of T cell anergy. Journal of Immunology 181 1622-1626.

Lineberry N, Su L, Soares L \& Fathman CG $2008 b$ The single-subunit transmembrane E3 ligase GRAIL captures and then ubiquitinates transmembrane proteins across the cell membrane. Journal of Biological Chemistry 283 28497-28505.

Matsumoto M, Ogawa W, Akimoto K, Inoue H, Miyake K, Furukawa K, Hayashi Y, Iguchi H, Matsuki Y, Hiramatsu R et al. 2003 PKC $\lambda$ in liver mediates insulin-induced SREBP-1c expression and determines both hepatic lipid content and overall insulin sensitivity. Journal of Clinical Investigation 112 935-944.

Miyake K, Ogawa W, Matsumoto M, Nakamura T, Sakaue H \& Kasuga M 2002 Hyperinsulinemia, glucose intolerance, and dyslipidemia induced by acute inhibition of phosphoinositide 3-kinase signaling in the liver. Journal of Clinical Investigation 110 1483-1491.

O'Brien RM, Streeper RS, Ayala JE, Stadelmaier BT \& Hornbuckle LA 2001 Insulin-regulated gene expression. Biochemical Society Transactions 29 552-558.

Okamoto Y, Ogawa W, Nishizawa A, Inoue H, Teshigawara K, Kinoshita S, Matsuki Y, Watanabe E, Hiramatsu R, Sakaue H et al. 2007 Restoration of glucokinase expression in the liver normalizes postprandial glucose disposal in mice with hepatic deficiency of PDK1. Diabetes 56 1000-1009.

Radziuk J \& Pye S 2001 Hepatic glucose uptake, gluconeogenesis and the regulation of glycogen synthesis. Diabetes/Metabolism Research and Reviews 17 250-272.

Reynolds A, Leake D, Boese Q, Scaringe S, Marshall WS \& Khvorova A 2004 Rational siRNA design for RNA interference. Nature Biotechnology 22 326-330.

Sano M, Tokudome S, Shimizu N, Yoshikawa N, Ogawa C, Shirakawa K, Endo J, Katayama T, Yuasa S, Ieda M et al. 2007 Intramolecular 
control of protein stability, subnuclear compartmentalization, and coactivator function of peroxisome proliferator-activated receptor gamma coactivator $1 \alpha$. Journal of Biological Chemistry 282 25970-25980.

Savage DB, Petersen KF \& Shulman GI 2007 Disordered lipid metabolism and the pathogenesis of insulin resistance. Physiological Reviews 87 507-520.

Seroogy CM, Soares L, Ranheim EA, Su L, Holness C, Bloom D \& Fathman CG 2004 The gene related to anergy in lymphocytes, an E3 ubiquitin ligase, is necessary for anergy induction in CD4 T cells Journal of Immunology 173 79-85.

Shimano H 2001 Sterol regulatory element-binding proteins (SREBPs): transcriptional regulators of lipid synthetic genes. Progress in Lipid Research 40 439-452.

Soares L, Seroogy C, Skrenta H, Anandasabapathy N, Lovelace P, Chung CD, Engleman E \& Fathman CG 2004 Two isoforms of otubain 1 regulate T cell anergy via GRAIL. Nature Immunology 5 45-54.

Taniguchi CM, Ueki K \& Kahn R 2005 Complementary roles of IRS-1 and IRS-2 in the hepatic regulation of metabolism. Journal of Clinical Investigation 115 718-727.

Teshigawara K, Ogawa W, Mori T, Matsuki Y, Watanabe E, Hiramatsu R, Inoue H, Miyake K, Sakaue H \& Kasuga M 2005 Role of Kruppel-like factor 15 in PEPCK gene expression in the liver. Biochemical and Biophysical Research Communications 327 920-926.

Trinh KY, O’Doherty RM, Anderson P, Lange AJ \& Newgard CB 1998 Perturbation of fuel homeostasis caused by overexpression of the glucose-6-phosphatase catalytic subunit in liver of normal rats. Journal of Biological Chemistry 273 31615-31620.

Valera A, Pujol A, Pelegrin M \& Bosch F 1994 Transgenic mice overexpressing phosphoenolpyruvate carboxykinase develop noninsulin-dependent diabetes mellitus. PNAS 91 9151-9154.

Yoon JC, Puigserver P, Chen G, Donovan J, Wu Z, Rhee J, Adelmant G, Stafford J, Kahn CR, Granner DK et al. 2001 Control of hepatic gluconeogenesis through the transcriptional coactivator PGC-1. Nature 413 131-138.

Zhang Y, Lee FY, Barrera G, Lee H, Vales C, Gonzalez FJ, Willson TM \& Edwards PA 2006 Activation of the nuclear receptor FXR improves hyperglycemia and hyperlipidemia in diabetic mice. PNAS 103 1006-1011.

Received in final form 13 November 2008

Accepted 25 November 2008

Made available online as an Accepted Preprint 25 November 2008 